\title{
Sialyl SSEA-1 Antigen Measurement
}

National Cancer Institute

\section{Source}

National Cancer Institute. SialyI SSEA-1 Antigen Measurement. NCI Thesaurus. Code C114223.

The determination of the amount of sialyI SSEA-1 antigen present in a sample. 